
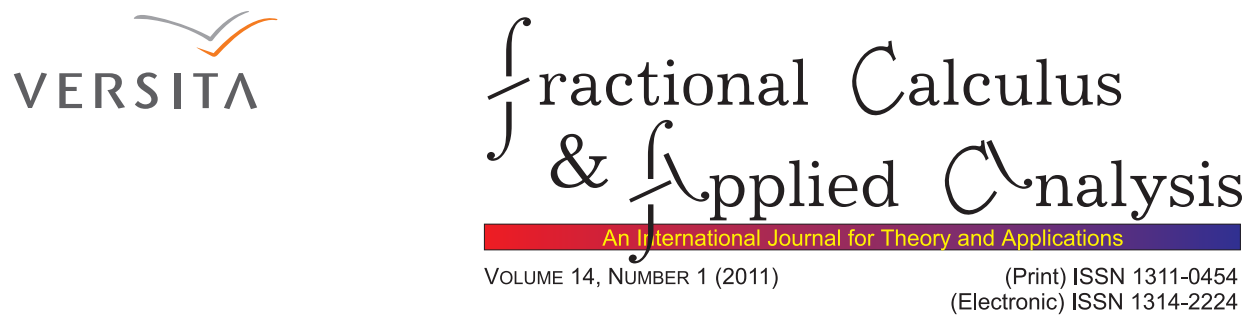

RESEARCH PAPER

\title{
HAMILTON'S PRINCIPLE WITH VARIABLE ORDER FRACTIONAL DERIVATIVES
}

\author{
Teodor M. Atanackovic ${ }^{1}$, Stevan Pilipovic ${ }^{2}$
}

\author{
Abstract \\ Dedicated to 80-th birthday of Prof. Rudolf Gorenflo
}

We propose a generalization of Hamilton's principle in which the minimization is performed with respect to the admissible functions and the order of the derivation. The Euler-Lagrange equations for such minimization are derived. They generalize the classical Euler-Lagrange equation. Also, a new variational problem is formulated in the case when the order of the derivative is defined through a constitutive equation. Necessary conditions for the existence of the minimizer are obtained. They imply various known results in a special cases.

MSC 2010: 26A33, 70H25, 46F12, 34K37

Key Words and Phrases: variable order fractional derivative, variational principle of Hamilton's type

\section{Introduction}

Hamilton's principle of least action is one of the fundamental principles of physics. According to it a system moves in such a way that the time integral over its Lagrangian takes an extreme value on the real path of the system when compared with the neighboring paths having the same end

(c) 2011 Diogenes Co., Sofia pp. $94-109$, DOI: $10.2478 / \mathrm{s} 13540-011-0007-7$ 
points. In this paper, that has mainly expository character, we present different approaches to the Hamilton's principle with variable order fractional derivatives, indicating our further work in this direction.

When Hamilton's principle is known, all the information regarding the processes of a particular system are included into its Lagrangian. There have been many generalizations of Hamilton's principle, see for example [25], [1] and references therein. Recently, the Hamiltons's principle has been generalized by replacing the integer order derivatives in Lagrangian with the fractional ones (derivatives of real order). The first study of Hamilton's principle with fractional derivatives was conducted by Riewe ([19]), ([20]) while for other works, we refer to [6], [16], [4].

Our intention in this work is to generalize the fractional Hamilton's principle by introducing variable order fractional derivative into the Lagrangian. Variable order fractional derivatives are presented in several publications (see for example [21], [15], 8, [23, [9] and [17]). In our work, we shall replace the integer order derivative in the Lagrangian density with the variable order fractional derivatives so that the action integral has the form

$$
I=\int_{t_{0}}^{t_{1}} L\left(t, y(t),{ }_{0} D_{t}^{\alpha(t)} y, \alpha(t)\right) d t
$$

where $L$ is the Lagrangian density, $y$ is a generalized coordinate, ${ }_{0} D y_{t}^{\alpha(t)}$ denotes the left Riemann-Liouville fractional derivative of the order $\alpha=$ $\alpha(t), t \in\left[t_{0}, t_{1}\right]$ of $y$ and $t_{0}$ and $t_{1}$ are given time instants. Our main assumption is that the minimization in (11) should be performed with respect to both $y$ and $\alpha$. We believe that this constitutes a novel approach to Hamilton's principle that allows determination of both $y$ and $\alpha$. Also this minimization leads to a new type of variational problem. To obtain the necessary conditions for the optimality in the case of Lagrangian having variable order fractional derivatives we need the corresponding integration by parts formula. In Section 4 we derive this formula for one (of several proposed) types of fractional derivatives of variable order.

We stress on the fact that our results depend on the definition of fractional derivative of variable order. In publications that appeared lately different definitions of variable order fractional derivatives have been introduced. The questions raised in these publications have a deep meaning from both mathematical and physical point of view (see [17], [8]). The integration by parts formula that we derive here and the resulting variational principles, show that the definition based on distributional approach to fractional derivatives has certain advantages among proposed definitions.

We recall the definitions of the fractional integrals of constant real $(\alpha=$ const) order. Let $t \in[0, T], T>0$. The left and right Riemann-Liouville 
(RL) fractional integrals are given as

$$
{ }_{0} I_{t}^{\alpha} y=\frac{1}{\Gamma(\alpha)} \int_{0}^{t}(t-\tau)^{\alpha-1} y(\tau) d \tau, \quad 0 \leq t \leq T,
$$

respectively

$$
{ }_{t} I_{T}^{\alpha} y=\frac{1}{\Gamma(\alpha)} \int_{t}^{T}(\tau-t)^{\alpha-1} y(\tau) d \tau, \quad 0 \leq t \leq T,
$$

Let $0<\alpha<1,1 \leq p, q<1$. Then the operators ${ }_{0} I_{t}^{\alpha} y$ and ${ }_{t} I_{T}^{\alpha} y$ are bounded and linear ones from $L_{p}([0, T])$ to $L_{q}([0, T])$ if $1<p<1 / \alpha, q=p / 1-\alpha p$ (see [22]).

The left and right RL fractional derivatives are defined as

$$
{ }_{0} D_{t}^{\alpha} y=\frac{d}{d t} \frac{1}{\Gamma(1-\alpha)} \int_{0}^{t} \frac{y(\tau)}{(t-\tau)^{\alpha}} d \tau, \quad 0 \leq t<T
$$

and

$$
{ }_{t} D_{T}^{\alpha} y=-\frac{d}{d t} \frac{1}{\Gamma(1-\alpha)} \int_{t}^{T} \frac{y(\tau)}{(\tau-t)^{\alpha}} d \tau, \quad 0 \leq t<T,
$$

respectively. If $y \in C^{1}[0, T]$, the derivatives ${ }_{0} D_{t}^{\alpha} y$ and ${ }_{t} D_{T}^{\alpha} y$ exist, belong to $L_{r}[0, T]$ and are given by the following expressions $(t \in[0, T])$

$$
\begin{aligned}
& { }_{0} D_{t}^{\alpha} y=\frac{y(0+)}{\Gamma(1-\alpha) t^{\alpha}}+\frac{1}{\Gamma(1-\alpha)} \int_{0}^{t} \frac{y^{(1)}(\tau)}{(t-\tau)^{\alpha}} d \tau, 0 \leq t \leq T, \\
& { }_{t} D_{T}^{\alpha} y=\frac{y(T)}{\Gamma(1-\alpha)(T-t)^{\alpha}}-\frac{1}{\Gamma(1-\alpha)} \int_{t}^{T} \frac{y^{(1)}(\tau)}{(\tau-t)^{\alpha}} d \tau, 0 \leq t \leq T,
\end{aligned}
$$

with $1 \leq r<1 / \alpha$.

Now suppose that $\alpha$ is not a constant but a given function of $t$, satisfying $0 \leq \alpha(t)<1, t \in[0, T]$. The problem is how to define ${ }_{0} I_{t}^{\alpha(t)} y,{ }_{0} D_{t}^{\alpha(t)} y,{ }_{t} I_{T}^{\alpha(t)} y$ and ${ }_{t} D_{T}^{\alpha(t)} y$. There are several definitions proposed in the literature. We state some of them. In all results that follow we assume $y(t)=0$ for $t<0$.

1) In 21] the following definition of left fractional integral of variable order is proposed

$$
{ }_{0} I_{t}^{\alpha(t)} y=\frac{1}{\Gamma(\alpha(t))} \int_{0}^{t}(t-\tau)^{\alpha(t)-1} y(\tau) d \tau, 0 \leq t \leq T
$$

2) In [15] several definitions are introduced. The first is identical to (66). The next one, is

$$
{ }_{0} I_{t}^{\alpha(t)} y=\int_{0}^{t} \frac{(t-\tau)^{\alpha(\tau)-1}}{\Gamma(\alpha(\tau))} y(\tau) d \tau, 0 \leq t \leq T .
$$


3) We state another definition proposed in [15], where it is assumed that $\alpha$ is a function of $(t-\tau)$, i.e.

$$
{ }_{0} I_{t}^{\alpha(t)} y=\int_{0}^{t} \frac{(t-\tau)^{\alpha(t-\tau)-1}}{\Gamma(\alpha(t-\tau))} y(\tau) d \tau, 0 \leq t \leq T .
$$

The fractional derivative of variable order could now be defined simply (as in the case of constant order) by taking the first derivative of the $(1-\alpha(t))$ integral given by any of the expressions (6) $-(8)$. This leads to the definition of the form

$$
{ }_{0} D_{t}^{\alpha(t)} y=\frac{d}{d t} \int_{0}^{t} \frac{(t-\tau)^{-q(t, \tau)}}{\Gamma(1-q(t, \tau))} y(\tau) d \tau,
$$

where $q(t, \tau)=\alpha(t), q(t, \tau)=\alpha(\tau)$ and $q(t, \tau)=\alpha(t-\tau)$, in cases (6) -(8). Thus, we obtain, respectively:

1)

$$
{ }_{0} D_{t}^{\alpha(t)}=\frac{d}{d t} \int_{0}^{t} \frac{(t-\tau)^{-\alpha(t)}}{\Gamma(1-\alpha(t))} y(\tau) d \tau, 0 \leq t \leq T,
$$

2)

$$
{ }_{0} D_{t}^{\alpha(t)} y=\frac{d}{d t} \int_{0}^{t} \frac{(t-\tau)^{-\alpha(\tau)}}{\Gamma(1-\alpha(\tau))} y(\tau) d \tau, 0 \leq t \leq T,
$$

3)

$$
{ }_{0} D_{t}^{\alpha(t)} y=\frac{d}{d t} \int_{0}^{t} \frac{(t-\tau)^{-\alpha(t-\tau)}}{\Gamma(1-\alpha(t-\tau))} y(\tau) d \tau, 0 \leq t \leq T .
$$

There are other definitions of variable order fractional integrals and derivatives. As will be seen, all definitions have, as a starting point, certain form of fractional derivative of constant order. Then, this form is generalized by allowing the constant $\alpha$ to become a function.

In [21] the Marchaud fractional derivative is used as a starting point, so that the variable order fractional derivative is defined as $(0<\alpha(t)<1)$ :

$$
{ }_{0} D_{t}^{\alpha(t)} y=\frac{y(t)}{\Gamma[1-\alpha(t)] t^{\alpha(t)}}+\frac{\alpha(t)}{\Gamma[1-\alpha(t)]} \int_{0}^{t} \frac{y(t)-y(\tau)}{(t-\tau)^{1+\alpha(t)}} d \tau, 0 \leq t \leq T .
$$

In [13] the following definition is proposed: 
$5)$

$$
\begin{aligned}
{ }_{0} D_{t}^{\alpha(t)} y & =\frac{d}{d t} \int_{0}^{t} \frac{y(t-\tau)}{\Gamma[1-\alpha(\tau)]^{\alpha(\tau)}} d \tau, \\
& =\frac{y(0)}{\Gamma(1-\alpha) t^{\alpha(t)}}+\frac{1}{\Gamma(1-\alpha)} \int_{0}^{t} \frac{y^{(1)}(\tau)}{(t-\tau)^{\alpha(\tau)}} d \tau, 0 \leq t \leq T,
\end{aligned}
$$

or alternatively

$$
{ }_{0} D_{t}^{\alpha(t)} y=\frac{d}{d t} \int_{0}^{t} \frac{y(\tau)}{\Gamma[1-\alpha(t-\tau)](t-\tau)^{\alpha(t-\tau)}} d \tau, 0 \leq t \leq T,
$$

which is (11).

We present now the definition given in [8]. As a motivation, note that starting from (4), assuming that $y \in A C([0, t])$ and that $\alpha=$ const, we obtain (see [22], p.32):

$$
{ }_{0} D_{t}^{\alpha(t)} y=\frac{y(0+)}{\Gamma(1-\alpha) t^{\alpha}}+\frac{1}{\Gamma(1-\alpha)} \int_{0}^{t} \frac{y^{(1)}(\tau)}{(t-\tau)^{\alpha}} d \tau, 0 \leq t \leq T,
$$

where $y^{(1)}(\tau)=d y(\tau) / d \tau$. By replacing $\alpha=$ const with $\alpha(t)$ in (15) it follows

$$
{ }_{0} D_{t}^{\alpha(t)} y=\frac{y(0)}{\Gamma(1-\alpha(t)) t^{\alpha(t)}}+\frac{1}{\Gamma(1-\alpha(t))} \int_{0}^{t} \frac{y^{(1)}(\tau)}{(t-\tau)^{\alpha(t)}} d \tau .0 \leq t \leq T .
$$

The definition proposed in [8] (see also [18]) reads:

$6)$

$$
{ }_{0} D_{t}^{\alpha(t)} y=\frac{y(0+)-y(0-)}{\Gamma(1-\alpha(t)) t^{\alpha(t)}}+\frac{1}{\Gamma(1-\alpha(t))} \int_{0}^{t} \frac{y^{(1)}(\tau)}{(t-\tau)^{\alpha(t)}} d \tau, 0 \leq t \leq T \text {. }
$$

For functions that satisfy $y(0)=0$ (since we assumed $y(t)=0$ for $t<0$ ) the definitions (16), (17) agree.

Next we present the definition of variable order fractional derivative based on Grünwald-Letnikov definition for derivative of constant order. This approach is especially useful in numerical treatment of differential equations with variable order derivatives. The left Grünwald-Letnikov fractional derivative of variable order (see [27]) is defined as:

7)

$$
{ }_{0} D_{t}^{\alpha(t)} y=\lim _{h \rightarrow 0, n h=t} h^{-\alpha(t)} \sum_{j=0}^{n}(-1)^{j}\left(\begin{array}{c}
\alpha(t) \\
j
\end{array}\right) y(t-j h) .
$$

It can be shown that in the case when $y$ is continuously differentiable (see [27]), then definitions (91) and (18) agree. 
The above definitions of variable order fractional derivatives are special cases of a general fractional variable order derivative proposed in [24] as:

$$
{ }_{0} D_{t}^{\alpha(t)} y=\frac{d}{d t} \int_{0}^{t} K_{\mu, \nu}^{\alpha(t)}(t, \tau) y(\tau) d \tau, 0 \leq t \leq T,
$$

where $\mu$ and $\nu$ are real parameters and

$$
K_{\mu, \nu}^{\alpha(t)}(t, \tau)=\frac{1}{\Gamma(1-\alpha(\mu t+\nu \tau))(t-\tau)^{\alpha(\mu t+\nu \tau)}}, \quad 0<\tau<t
$$

The parameters $\mu$ and $\nu$ belong to the causality parallelogram of LorenzoHartley $(\mu, \nu) \in \Pi$, where

$$
\Pi=\left\{(\mu, \nu) \in \mathbb{R}^{2}: 0 \leq \mu \leq 1,-1 \leq \nu \leq 1,0 \leq \mu+\nu \leq 1\right\} .
$$

Thus, for example with $\mu=0, \nu=1$, we recover (9) while with $\mu=1, \nu=$ -1 , (19) becomes (11).

As it is stated in [18] and [9, the problem of choosing a "proper" definition of variable order fractional derivative is open. In the next section we present an analysis which supports the definition given in (11).

\section{Distributional setting of variable order derivatives}

In this section we review within the theory of distributions the definition of the fractional derivative of constant order and propose a generalization to the case when the order of the derivative is also a function.

Let $\mathcal{S}(\mathbb{R})$ denote the space of rapidly decreasing functions in $\mathbb{R}$ (functions $\varphi$ is in $\mathcal{S}(\mathbb{R})$ if $\varphi(t)$ and all its derivatives tend to zero as $t \rightarrow \pm \infty$ faster than the inverted value of every polynomial) and let $\mathcal{S}^{\prime}(\mathbb{R})$ be its dual, i.e., the space of tempered distributions; $\mathcal{S}_{+}^{\prime}(\mathbb{R})$ denotes its subspace consisting of distributions supported by $[0, \infty)$. We consider in $\mathcal{S}_{+}^{\prime}(\mathbb{R})$ the family

$$
f_{\alpha}(t)=\left\{\begin{array}{cc}
H(t) \frac{t^{\alpha-1}}{\Gamma(\alpha)}, & t \in \mathbb{R}_{+}, \alpha>0, \\
\frac{d^{N}}{d t^{N}} f_{\alpha+N}(t), & \alpha \leq 0, \alpha+N>0, N \in \mathbb{N},
\end{array}\right.
$$

where $H$ is Heaviside's function and $\Gamma$ is the Euler Gamma function. It is well known that $f_{\alpha} * f_{\beta}=f_{\alpha+\beta}, \alpha, \beta \in \mathbb{R}$, where $*$ denotes the convolution of $f_{\alpha}$ and $f_{\beta}$. The convolution operator $f_{\alpha} *$ in $\mathcal{S}_{+}^{\prime}(\mathbb{R})$ is the operator of fractional differentiation for $\alpha<0$ and of fractional integration for $\alpha>0$. It coincides with the operator of derivation for $-\alpha \in \mathbb{N}$ and integration for $\alpha \in \mathbb{N}_{0}=\mathbb{N} \cup\{0\}$. Note $f_{\alpha=0}(t)=\delta(t)$, where $\delta$ is the Dirac Distribution. Let $\alpha>0$ and $y \in L_{\text {loc }}^{1}(\mathbb{R})$ be polynomially bounded with $y(t)=0$ for 
$t<0$. Then $I^{\alpha} y=f_{\alpha} * y$. In general, for $y \in \mathcal{S}_{+}^{\prime}(\mathbb{R})$ the fractional derivative of order $\alpha \in[0,1)$ is defined as

$$
{ }_{0} D_{t}^{\alpha} y=f_{-\alpha} * y=\frac{d}{d t} f_{-\alpha+1} * y,
$$

where

$$
f_{-\alpha}=\frac{d}{d t} H(t) \frac{t^{-\alpha}}{\Gamma(1-\alpha)},
$$

so that

$$
{ }_{0} D_{t}^{\alpha} y=\int_{0}^{t} \frac{d}{d \tau} \frac{\tau^{-\alpha}}{\Gamma(1-\alpha)} y(t-\tau) d \tau=\frac{d}{d t} \frac{1}{\Gamma(1-\alpha)} \int_{0}^{t} \frac{y(\tau)}{(t-\tau)^{\alpha}} d \tau .
$$

In [3] we proved that $y \longmapsto_{0} D_{t}^{\alpha} y$ is linear and continuous mapping from $\mathcal{S}_{+}^{\prime}(\mathbb{R})$ to $\mathcal{S}_{+}^{\prime}(\mathbb{R})$.

Suppose that $\alpha \in C^{1}([0, \infty)), 0 \leq \alpha(t)<1$. We consider a family in $\mathcal{S}_{+}^{\prime}(\mathbb{R})$ given by

$$
\left(f_{-\alpha(t)}(t)\right)_{+}=\frac{d}{d t} H(t) \frac{t^{\alpha(t)}}{\Gamma(1-\alpha(t))}, \quad t \in \mathbb{R}_{+}
$$

(see [9]). Let $\phi \in \mathcal{S}(\mathbb{R})$. Recall, the action of $\left(f_{-\alpha(t)}(t)\right)_{+}$on $\phi$ is $\left\langle f_{-\alpha(t)}(t), \phi\right\rangle$ $=-\left\langle f_{1-\alpha(t)}(t), \phi^{(1)}\right\rangle$. Thus, $\left(f_{-\alpha(t)}(t)\right)_{+}$is a tempered distribution.

With (23) and (25) we define the left variable order fractional RL integral and derivative as:

Definition 2.1. Suppose that $\alpha \in C[0, \infty), 0 \leq \alpha(t)<1$ and $y(t)=$ 0 for $t<0$. The left RL fractional integral of variable order $\alpha(t)$ is defined as

$$
{ }_{0} I_{t}^{\alpha(t)} y=\left(f_{-\alpha(t)}(t)\right)_{+} * y=\int_{0}^{t} \frac{y(\tau)}{\Gamma(\alpha(t-\tau))(t-\tau)^{1-\alpha(t-\tau)}} d \tau t \in \mathbb{R},
$$

and the left RL fractional derivative of variable fractional order $\alpha(t)$ is

$$
{ }_{0} D_{t}^{\alpha(t)} y=f_{-\alpha(t)} * y=\frac{d}{d t} \int_{0}^{t} \frac{(t-\tau)^{-\alpha(t-\tau)}}{\Gamma(1-\alpha(t-\tau))} y(\tau) d \tau, t \in \mathbb{R}
$$

In (27) the derivative is taken in the sense of distributions. Note that ${ }_{0} D_{t}^{\alpha(t)} y=\frac{d}{d t}{ }_{0} I_{t}^{1-\alpha(t)}$. Also if $y \in \mathcal{S}_{+}^{\prime}(\mathbb{R})$, then ${ }_{0} I_{t}^{\alpha(t)} y \in \mathcal{S}_{+}^{\prime}(\mathbb{R})_{0}$ and $D_{t}^{\alpha(t)} y \in \mathcal{S}_{+}^{\prime}(\mathbb{R})$ (since $f_{-\alpha}(t), 0 \leq \alpha(t)<1$ is a locally integrable function). The definitions reduce to the classical ones when $\alpha=$ const. From (27) the assumption of [15] that the arguments of $\alpha$ in the exponent of $(t-\tau)$ and in the gamma function, of equation (11) are the same, here follows naturally. Physically, definition (26) and (27) imply that a memory of the value of 
order of integration $\alpha$ is taken into account in the evaluation of a fractional derivative. Based on (27), we define for absolutely continuous functions fractional derivatives as follows:

Definition 2.2. Let $\alpha \in C[0, T), 0 \leq \alpha(t)<1$ and $y \in A C([0, T])$. Then left and right RL fractional derivatives of variable order $\alpha$ are defined as:

$$
{ }_{0} D_{t}^{\alpha(t)} y=\frac{y(0)}{\Gamma(1-\alpha(t)) t^{\alpha(t)}}+\int_{0}^{t} \frac{y^{(1)}(\tau)}{\Gamma(1-\alpha(t-\tau))(t-\tau)^{\alpha(t-\tau)}} d \tau,
$$

and

$$
{ }_{t} D_{T}^{\alpha(t)} y=\frac{y(T)}{\Gamma(1-\alpha(T-t)) t^{\alpha(T-t)}}-\int_{t}^{T} \frac{y^{(1)}(\tau)}{\Gamma(1-\alpha(\tau-t))(\tau-t)^{\alpha(\tau-t)}} d \tau,
$$

respectively.

\section{Integration by parts formula}

In this section we consider the integration by parts formula ([14], p.76) for fractional derivatives of variable order. Thus, let $\alpha \in C[0, T), 0 \leq$ $\alpha(t)<1, y(t)=0$ for $t<0$ and $y(t)=0$ for $t>T$. Recall that, for $\alpha=$ const. and $0<\alpha<1$ we have: If $f \in{ }_{0} I_{t}^{\alpha}\left(L_{p}[0, T]\right)$ and $g \in{ }_{t} I_{T}^{\alpha}\left(L_{q}[0, T]\right)$, $1 / p+1 / q \leq 1+\alpha$, then

$$
\int_{0}^{T}{ }_{0} D_{t}^{\alpha} f(t) g(t) d t=\int_{0}^{T} f(t){ }_{t} D_{T}^{\alpha} g(t) d t,
$$

where ${ }_{0} I_{t}^{\alpha}\left(L_{p}[0, T]\right)$ and ${ }_{t} I_{T}^{\alpha}\left(L_{q}[0, T]\right)$ denote the class of functions $f$ and $g$ that can be represented as Riemann-Liouville integrals, that is $f={ }_{0} I_{t}^{\alpha} \varphi$ for some $\varphi \in L_{p}[0, T]$ and $g={ }_{t} I_{T}^{\alpha} \psi$ for some $\psi \in L_{q}[0, T]$, respectively. Note that for the case when $\alpha \rightarrow 1$ the equation (30) reduces to the known result.

For variable order fractional derivative given by (28),(29) we have the following:

Proposition 3.1. Suppose that $y \in A C([0, T]), 0 \leq \alpha(t)<1, t \in$ $[0, T]$. Then the following integration by parts formula holds:

$$
\int_{0}^{T} f(t)_{a} D_{t}^{\alpha(t)} y(t) d t=\int_{0}^{T} y(t)_{t} D_{T}^{\alpha(t)} f(t) d t
$$


To prove the proposition, we transform the left hand side term

$$
\int_{0}^{T} f(t)_{0} D_{t}^{\alpha(t)} y(t) d t
$$

as follows:

$$
\begin{gathered}
\int_{0}^{T} f(t)_{0} D_{t}^{\alpha(t)} y(t) d t=\int_{0}^{T} f(t)\left[\frac{d}{d t} \int_{0}^{t} \frac{y(\tau)}{\Gamma(1-\alpha(t-\tau))(t-\tau)^{\alpha(t-\tau)}} d \tau\right] d t \\
\left.=\left.\left.f(t) \int_{0}^{t} \frac{y(\tau)}{\Gamma(1-\alpha(t-\tau))(t-\tau)^{\alpha(t-\tau)}} d \tau\right|_{t=0} ^{t=T}\right|_{0} ^{t} \frac{y(\tau)}{\Gamma(1-\alpha(t-\tau))(t-\tau)^{\alpha(t-\tau)}} d \tau\right] d t \\
=f(T)\left[\int_{0}^{T} \frac{\int_{0}^{(1)}(t)\left[\int_{0}^{t} \frac{f^{(1)}(t)}{\Gamma(1-\alpha(T-\tau))(T-\tau)^{\alpha(T-\tau)}} d \tau\right]}{\int_{0}^{T} y(\tau)\left[\int_{\tau}^{T} \frac{f(T)}{\Gamma(1-\alpha(t-\tau))(t-\tau)^{\alpha(t-\tau)}} d t\right] d \tau}\right. \\
=\int_{0}^{T} y(\tau)\left[\frac{f^{(1)}(t)}{\Gamma(1-\alpha(T-\tau))(T-\tau)^{\alpha(b-\tau)}}\right. \\
\left.\int_{\tau}^{T} \frac{\int^{\alpha}}{\Gamma(1-\alpha(t-\tau))(t-\tau)^{\alpha(t-\tau)}} d t\right] d \tau \int_{0}^{T} y(t)_{t} D_{T}^{\alpha(t)} f(t) d t .
\end{gathered}
$$

Thus, we conclude that the definition (28), (29) preserves the form of integration by parts formula (30). The expression (31) is the basis for Variational principle with Lagrangian having derivatives of variable order that we treat in the next section.

\section{Variational principle for variable order fractional derivative: Generalized Hamilton's principle}

Consider the Lagrangian density be given by

$$
L(y, \alpha)=L\left(t, y(t),{ }_{a} D_{t}^{\alpha(t)} y(t), \alpha(t)\right)
$$

with $y$ and $\alpha$ belonging to specified spaces. We consider the problem of minimizing the following functional

$$
I=\int_{0}^{T} L\left(t, y(t),{ }_{a} D_{t}^{\alpha(t)} y(t), \alpha(t)\right) d t .
$$

Here we assume that $L$ depends on time, generalized coordinate, fractional derivative of generalized coordinate and order of the derivative function. Also $y \in \mathcal{U}$, where $\mathcal{U}$ is the space of admissible functions and $\alpha \in \mathcal{V}$ where 
$\mathcal{V}$ is the space of admissible order functions. In each specific example $\mathcal{U}$ and $\mathcal{V}$ are specified in advance. Generally, $\mathcal{U}$ will be the set of functions satisfying some regularity properties (for example $y \in A C([0, T])$ ) and specified boundary conditions. For simplicity we assume again that $0<$ $\alpha(t)<1, t \in[0, T]$.

We shall distinguish the following cases:

1) Function $\alpha$ is given. The problem then becomes: Find $y=y^{*}$ such that

$$
\begin{aligned}
\min _{y \in \mathcal{U}} I(y, \alpha) & =\min _{y \in \mathcal{U}} \int_{0}^{T} L\left(t, y(t),{ }_{0} D_{t}^{\alpha(t)} y(t), \alpha(t)\right) d t \\
& =\int_{0}^{T} L\left(t, y^{*}(t),{ }_{0} D_{t}^{\alpha(t)} y^{*}(t), \alpha(t)\right) d t .
\end{aligned}
$$

To obtain necessary conditions for the minimum, we assume that $y=y^{*}+\varepsilon h$ where $\varepsilon \in \mathbb{R}$, and $h$ is such that $y \in \mathcal{U}$ for all $\varepsilon$. Since $\alpha$ is given it is not subject to variation. By substituting (33) into (32) we find that for each $h$

$$
I\left(y^{*}+\varepsilon h, \alpha\right)=\int_{0}^{T} L\left(t, y^{*}(t)+\varepsilon h(t),{ }_{0} D_{t}^{\alpha(t)}\left(y^{*}(t)+\varepsilon h(t)\right), \alpha(t)\right) d t,
$$

is a function of $\varepsilon$ only. Thus $\frac{d}{d \varepsilon}\left(I\left(y^{*}+\varepsilon h, \alpha\right)\right)_{\varepsilon=0}=0$ leads to

$$
\int_{0}^{T}\left[\frac{\partial L}{\partial y} h(t)+\frac{\partial L}{\partial_{a} D_{t}^{\alpha(t)} y}{ }_{a} D_{t}^{\alpha(t)} h(t)\right] d t=0 .
$$

Using (31) we obtain the necessary condition for the minimum of (32) in the form of Euler-Lagrange equation

$$
\frac{\partial L}{\partial y}+{ }_{t} D_{T}^{\alpha(t)} \frac{\partial L}{\partial_{a} D_{t}^{\alpha(t)} y}=0 .
$$

Equation (35) is a generalization of the classical Euler-Lagrange equations as well as generalization of the Euler-Lagrange equations given in [6] for $\alpha=$ const.

2) Function $\alpha$ is a constant, i.e., $\alpha=$ const such that $\alpha \in[0,1)$ but is not given in advance. Then minimization of (32) becomes

$$
\left.\min _{y \in \mathcal{U}, \alpha \in[0,1]} I(y, \alpha)=\min _{y \in \mathcal{U}, \alpha \in[0,1)} \int_{0}^{T} L\left(t, y(t),{ }_{0} D_{t}^{\alpha} y(t), \alpha\right)\right) d t .
$$

The necessary conditions for optimality of (36) read (see [5])

$$
\frac{\partial L}{\partial y}+{ }_{t} D_{T}^{\alpha} \frac{\partial L}{\partial_{0} D_{t}^{\alpha} y}=0, \int_{0}^{T}\left(\frac{\partial L}{\partial_{0} D_{t}^{\alpha} y} G(y, \alpha)+\frac{\partial L}{\partial \alpha}\right) d t=0,
$$


where

$G(y, \alpha)=\frac{\partial_{0} D_{t}^{\alpha} y}{\partial \alpha}=\frac{d}{d t}\left(f_{1} * y\right)(t, \alpha), \quad f_{1}(t, \alpha)=\frac{1}{t^{\alpha} \Gamma(1-\alpha)}[\psi(1-\alpha)-\ln t]$,

$t>0$, with the Euler function $\psi(z)=\frac{d}{d z} \ln \Gamma(z)$, and $\left(f_{1} * y\right)(t, \alpha)=$ $\int_{0}^{t} f_{1}(\tau, \alpha) y(t-\tau) d \tau$. The expression (38) holds for $y$ being locally integrable function, i.e., $y \in L_{\text {loc }}^{1}(\mathbb{R})$ with $y(t)=0$ for $t<0$. In [5] the expression for $\frac{\partial_{0} D_{t}^{\alpha} y}{\partial \alpha}$ is determined also for the case when $y \in S_{+}^{\prime}(\mathbb{R})$ and consequently ${ }_{0} D_{t}^{\alpha} y \in S_{+}^{\prime}(\mathbb{R})$, where $S_{+}^{\prime}(\mathbb{R})$ denotes the subspace of $S^{\prime}(\mathbb{R})$ consisting of tempered distributions supported by $[0, \infty)$. In this case (see [5, p.604)

$$
\begin{aligned}
\left\langle\frac{\partial_{0} D_{t}^{\alpha} y}{\partial \alpha}, \phi(t)\right\rangle & =\frac{\partial}{\partial \alpha}\left\langle\frac{d}{d t}{ }_{0} I_{t}^{1-\alpha} y, \phi(t)\right\rangle=-\frac{\partial}{\partial \alpha}\left\langle{ }_{0} I_{t}^{1-\alpha} y, \phi^{(1)}\right\rangle= \\
& =-\left\langle\frac{\partial}{\partial \alpha}{ }_{0} I_{t}^{1-\alpha} y, \phi^{(1)}\right\rangle=\left\langle\frac{d}{d t} \frac{\partial}{\partial \alpha}{ }_{0} I_{t}^{1-\alpha} y, \phi\right\rangle \\
& \left.=\left\langle\frac{d}{d t}\left(f_{1} * y\right)(t, \alpha)\right), \phi\right\rangle
\end{aligned}
$$

where $\phi \in \mathcal{S}(\mathbb{R})$ and we used the fact (see Section 2) that $\frac{\partial}{\partial \alpha}{ }_{0} I_{t}^{1-\alpha} y=$ $\left(f_{1} * y\right)(t, \alpha)$ with $f_{1}$ given by the second part of $(38)$.

3) Function $0<\alpha(t)<1, t \in[0, T]$, is not given and must be determined form the minimization of (32). Thus, we have

$$
\min _{y \in \mathcal{U}, \alpha \in \mathcal{V}} I(y, \alpha)=\min _{y \in \mathcal{U}, \alpha \in \mathcal{V}} \int_{0}^{T} L\left(t, y(t),{ }_{a} D_{t}^{\alpha(t)} y(t), \alpha(t)\right) d t
$$

Let $y=y^{*}$ and $\alpha=\alpha^{*}$ be the functions such that

$$
\min _{y \in \mathcal{U}, \alpha \in \mathcal{V}} \int_{0}^{T} L\left(t, y(t),{ }_{a} D_{t}^{\alpha(t)} y(t)\right) d t=\int_{0}^{T} L\left(t, y^{*}(t){ }_{a} D_{t}^{\alpha^{*}(t)} y^{*}(t), \alpha\right) d t
$$

To obtain the necessary conditions for the optimality in the problem (41) we let $y=y^{*}+\varepsilon_{1} h, \alpha=\alpha^{*}+\varepsilon_{2} \theta$ and $\theta$ such that $y \in \mathcal{U}, \alpha \in \mathcal{V}$ for all $\varepsilon_{1}, \varepsilon_{2} \in \mathbb{R}$. Again substituting this into $\int_{0}^{T} L\left(t, y(t),{ }_{a} D_{t}^{\alpha} y(t)\right) d t$ and by observing that there is no relation between $y$ and $\alpha$ we obtain (see [10]) the 
following necessary condition for optimality

$$
\begin{array}{r}
\int_{0}^{T}\left(\frac{\partial L}{\partial y}+{ }_{t} D_{T}^{\alpha(t)} \frac{\partial L}{\partial_{0} D_{t}^{\alpha(t)} y}\right) h(t) d t=0, \\
\int_{0}^{T}\left(\frac{\partial L}{\partial_{0} D_{t}^{\alpha(t)} y} \frac{\partial_{0} D_{t}^{\alpha(t)} y}{\partial \alpha(t)}+\frac{\partial L}{\partial \alpha(t)}\right) \theta(t) d t=0 .
\end{array}
$$

Now, by using (38), it follows that

$$
\begin{array}{r}
\int_{0}^{T}\left(\frac{\partial L}{\partial y}+{ }_{t} D_{T}^{\alpha(t)} \frac{\partial L}{\partial_{0} D_{t}^{\alpha(t)} y}\right) h(t) d t=0, \\
\int_{0}^{T}\left(\frac{\partial L}{\partial_{0} D_{t}^{\alpha(t)} y} Q(y, \alpha)+\frac{\partial L}{\partial \alpha(t)}\right) \theta(t) d t=0,
\end{array}
$$

where $Q(y, \alpha)=\frac{\partial_{0} D_{t}^{\alpha(t)} y}{\partial \alpha(t)}$. Under suitable conditions, (see [10], p. 9-11) we obtain from (43)

$$
\frac{\partial L}{\partial y}+{ }_{t} D_{b}^{\alpha} \frac{\partial L}{\partial_{0} D_{t}^{\alpha} y}=0, \frac{\partial L}{\partial_{0} D_{t}^{\alpha} y} Q(y, \alpha)+\frac{\partial L}{\partial \alpha}=0 .
$$

To determine $Q(y, \alpha)$ we proceed as in the case (38), (39) so that

$$
\begin{aligned}
& Q(y, \alpha)=\frac{\partial_{0} D_{t}^{\alpha(t)} y}{\partial \alpha(t)}=\frac{d}{d t}\left(g_{1} * y\right)(t, \alpha), \\
& g_{1}(t, \alpha)=\frac{1}{t^{\alpha} \Gamma(1-\alpha(t))}[\psi(1-\alpha(t))-\ln t], \quad t>0,
\end{aligned}
$$

and

$$
\left.\left\langle\frac{\partial_{0} D_{t}^{\alpha} y}{\partial \alpha}, \phi(t)\right\rangle=\left\langle\frac{d}{d t}\left(g_{1} * y\right)(t, \alpha)\right), \phi\right\rangle
$$

for $y \in L_{l o c}^{1}(\mathbb{R})$ and $y \in S_{+}^{\prime}(\mathbb{R})$, respectively.

4) Function $\alpha$ is not given explicitly, but is given in terms of $t, \alpha$ and $y$ by an additional differential equation equation. Thus, the optimization problem is formulated as: Minimize

$$
\min _{y \in \mathcal{U}} I(y, \alpha)=\int_{0}^{T} L\left(t, y(t),{ }_{a} D_{t}^{\alpha(t)} y(t), \alpha(t)\right) d t
$$

subject to

$$
\frac{d \alpha}{d t}=\Psi(t, \alpha(t), y(t)) .
$$


This situation is typical for internal variable theories. Namely, $\alpha$ is treated in (47) as an internal variable that is determined through constitutive equation (48). Thus, we have to solve

$$
\min _{y \in \mathcal{U}} I(y, \alpha)=\min _{y \in \mathcal{U}} \int_{0}^{T} L\left(t, y(t),{ }_{a} D_{t}^{\alpha(t)} y(t), \alpha(t)\right) d t
$$

subjected to the constraint (48). We rewrite (49),(48) as: find a minimum of

$$
\min _{y \in \mathcal{U}, z \in \mathcal{V}}(y, z, \alpha)=\min _{y \in \mathcal{U}, z \in \mathcal{V}} \int_{0}^{T} L(t, y(t), z(t), \alpha(t)) d t
$$

where $\mathcal{U}$, and $\mathcal{V}$ are given, subjected to the constraints

$$
\frac{d \alpha}{d t}=\Psi(\tau, \alpha(t), y(t)), \quad{ }_{a} D_{t}^{\alpha(t)} y(t)=z(t) .
$$

Using the Lagrange multiplier rule (see [6], [7], [12]) we consider the modified functional

$$
\begin{gathered}
\min _{y \in \mathcal{U}} \bar{I}(y, z, \alpha)=\min _{y \in \mathcal{U}} \int_{0}^{T}\{L(t, y(t), z(t), \alpha(t)) \\
\left.+p\left[z(t)-{ }_{a} D_{t}^{\alpha(t)} y(t)\right]+q\left[\Psi(\tau, \alpha(t), y(t))-\alpha^{(1)}\right]\right\} d t,
\end{gathered}
$$

where $p$ and $q$ are Lagrange multipliers. The necessary condition for the optimality $\delta \bar{I}=0$, after partial integration and the use of (31), becomes

$$
\begin{aligned}
\delta \bar{I} & =\int_{0}^{T}\left\{\left[\frac{\partial L}{\partial y}-{ }_{t} D_{1}^{\alpha(t)} p+q \frac{\partial \Psi}{\partial y}\right] \delta y\right. \\
& +\left[z-{ }_{a} D_{t}^{\alpha(t)} y(t)\right] \delta p+\left[\Psi(\tau, \alpha(t), y(t))-\alpha^{(1)}\right] \delta q+\left[\frac{\partial L}{\partial z}+p\right] \delta z \\
& \left.+\left[\frac{\partial L}{\partial \alpha}-p \frac{\partial_{a} D_{t}^{\alpha(t)}}{\partial \alpha(t)}+q^{(1)}(t)\right] \delta \alpha\right\} d t
\end{aligned}
$$

Now, from (53) we obtain

$$
\begin{gathered}
\alpha^{(1)}=\Psi(\tau, \alpha(t), y(t)), \quad{ }_{a} D_{t}^{\alpha(t)} y(t)=z(t), \\
{ }_{t} D_{1}^{\alpha(t)} p=\frac{\partial L}{\partial y}+q \frac{\partial \Psi}{\partial y}, \quad q^{(1)}(t)=-\frac{\partial L}{\partial \alpha}+p \frac{\partial_{a} D_{t}^{\alpha(t)}}{\partial \alpha(t)}, \quad p=-\frac{\partial L}{\partial z},
\end{gathered}
$$

with boundary conditions on $p$ and $q$ depending on the boundary conditions on $\alpha$ and $z$. Thus, for example, if $\alpha(0)$ and $\alpha(1)$ are arbitrary, we have $q(0)=q(1)=0($ see $[26])$. 
REMARK 4.1. Condition (54) implies the following special cases: If $\alpha=$ const that is prescribed, then $\Psi(\tau, \alpha(t), y(t))=0$ and since $\delta \alpha(0)=$ $\delta \alpha(1)=0$ we have $\delta q(0)$ and $\delta q(1)$ arbitrary. From (54) we then obtain

or

$$
{ }_{a} D_{t}^{\alpha(t)} y(t)=z(t),{ }_{t} D_{1}^{\alpha(t)} p=\frac{\partial L}{\partial y}, \quad p=-\frac{\partial L}{\partial z},
$$

$$
\frac{\partial L}{\partial y}+{ }_{t} D_{1}^{\alpha} \frac{\partial L}{\partial_{0} D_{t}^{\alpha} y}=0
$$

the result (56) obtained in [6]. If we assume that $\alpha=$ const but is not given in advance, we have $\Psi(\tau, \alpha(t), y(t))=0$ and $\delta \alpha(0)$ and $\delta \alpha(1)$ arbitrary. This implies $q(0)=q(1)=0$. From (54) we obtain

$$
\begin{aligned}
& \alpha^{(1)}=0, \quad{ }_{a} D_{t}^{\alpha(t)} y(t)=z(t),{ }_{t} D_{1}^{\alpha(t)} p=\frac{\partial L}{\partial y}, \\
& q(t)=\int_{0}^{t}\left[-\frac{\partial L}{\partial \alpha}-\frac{\partial L}{{ }_{a} D_{t}^{\alpha} y(t)} \frac{\partial_{a} D_{t}^{\alpha}}{\partial \alpha(t)}\right] d t, p=-\frac{\partial L}{\partial z},
\end{aligned}
$$

or

$$
\begin{array}{r}
\frac{\partial L}{\partial y}+{ }_{t} D_{b}^{\alpha} \frac{\partial L}{\partial_{0} D_{t}^{\alpha} y}=0 \\
\int_{0}^{1}\left[-\frac{\partial L}{\partial \alpha}-\frac{\partial L}{{ }_{a} D_{t}^{\alpha} y(t)} \frac{\partial_{a} D_{t}^{\alpha}}{\partial \alpha}\right] d t=0
\end{array}
$$

where we used the condition $q(1)=0$. The result (57) was obtained recently in [5].

\section{Conclusion}

In this work we recalled several definitions of fractional derivative of variable order. We concluded that definitions based on generalized distributional definition of left (11) and left and right fractional derivative of variable order (27) preserve the integration by parts formula. This enabled us to formulate several variational principles (optimization problems) listed as problems 1), 2) and 3) in Section 4. These variational principles may be used to determine differential equations of process together with order function $\alpha$ as a constant (Case 2) or as a function (Case 3). Also, we proposed a new optimization problem (Case 4) in which $\alpha$ is determined as a constitutive quantity. Central results on which our analysis is based are relations (38) and its generalization (45). We believe that new variational problem (47), (48) may be used to give rational basis for obtaining equations of process when the order of the derivative changing with time and value of the dependent (state) variable. An example of this type is presented in [11] where the order of the derivative was taken to be defined as 


$$
\alpha(t)=\frac{1+y^{2}(t)}{2} .
$$

Our analysis (equation (48)) can be easily modified to include (58) as a constraint.

\section{References}

[1] T.M. Atanackovic, On a stationarity principle for non conservative dynamical systems. Int. J. Non-Linear Mechanics 13 (1978), 139-143.

[2] T.M. Atanackovic, Lj. Oparnica and S. Pilipovic, On a nonlinear distributed order fractional differential equation. J. Math. Anal. Appl. 328 (2007), 590-608.

[3] T.M. Atanackovic, Lj. Oparnica, S. Pilipovic, Distributional framework for solving fractional differential equations. Integral Transf. Spec. Funct. 20 (2009), 215-222.

[4] T.M. Atanackovic, S. Konjik, S. Pilipovic, Variational problems with fractional derivatives: Euler-Lagrange equations. J. Phys. A: Math. Theor. 41 (2008), 095201.

[5] T.M. Atanackovic, S. Konjik, Lj. Oparnica, S. Pilipovic, Generalized Hamilton's principle with fractional derivatives. J. Phys. A: Math. Theor. 43 (2010), 255203.

[6] O.P. Agrawal, Formulation of Euler-Lagrange equations for fractional variational problems. J. Math. Anal. Appl. 272, 368-379 (2002).

[7] O. P. Agrawal, A general formulation and solution scheme for fractional optimal control problems, Nonlinear Dynamics 38 (2004), 323-337.

[8] C.F.M. Coimbra, Mechanics with variable-order differential operators. Ann. Phys. (Leipzig) 12 (2003), 692-703.

[9] S. Das, Functional Fractional Calculus. Springer, Berlin, 2008.

[10] I.M. Gelfand, S.V. Fomin, Calculus of Variations. Prentice Hall, Englewood Cliffs, New Jersey, 1963.

[11] G. Diaz, C.F.M. Coimbra, Nonlinear dynamics and control of a variable order oscillator with application to the van der Pol equation. Nonlinear Dyn. 56 (2009), 145-157.

[12] K. Ito, K. Kunisch, Lagrange Multiplier Approach to Variational Problems and Applications. Ser. SIAM's Advances in Design and Control, 2008.

[13] D. Ingman, J. Suzdalnitsky, Control of damping oscillations by fractional differential operator with time-dependent order. Comput. Methods Appl. Mech. Engrg. 193 (2004), 5585-5595.

[14] A. Kilbas, H.M. Srivastava, J.J. Truillo, Theory and applications of Fractional Differential Equations. Elsevier, 2006. 
[15] C.F. Lorenzo and T.T. Hartley, Variable order and distributed order fractional operators. Nonlin. Dyn. 29 (2002), 57-98.

[16] S.I. Muslih, D. Baleanu, Hamiltonian formulation of systems with linear velocities within Riemann-Liouville fractional derivatives. J. Math. Anal. Appl. 304 (2005), 599-606.

[17] L.E.S. Ramirez, C.F.M. Coimbra, A variable order constitutive relation for viscoelasticity. Ann. Phys. (Leipzig) 16 (2007), 543-552.

[18] L.E.S. Ramirez, C.F.M.Coimbra, On the selection and meaning of variable order operators for dynamic modeling. Intern. Journal of Differential Equations, To appear (2010), doi:10.1155/2010/846107.

[19] F. Riewe,Nonconservatice Lagrangian and Hamiltonian mechanics. Physical Review E 53 (1996), 1890-1899.

[20] F. Riewe, Mechanics with fractional derivatives. Physical Review E 55 (1997), 3581-3592.

[21] B. Ross, S. Samko, Integration and differentiation to a variable fractional order. Integr. Transf. Spec. Funct., 1 (1993), 277-300.

[22] S.G. Samko, A.A. Kilbas, O.I. Marichev, Fractional Integrals and Derivatives: Theory and Applications. Gordon and Breach, Amsterdam, 1993.

[23] C.M. Soon, C.F.M. Coimbra, M.H. Kobayashi, The variable viscoelasticity oscillator. Ann. Phys. (Leipzig) 14 (2005), 378-389.

[24] S. Umarov, S. Steinberg, Variable order differential equations with piecewise constant order-function and diffusion with changing modes. Zeitschrift für Analysis und iher Anwendungen 28 (2009), 431-450.

[25] B.D. Vujanovic, S.E. Jones, Variational Methods in Nonconservative Phenomena. Academic Press, Boston, 1989.

[26] B.D. Vujanovic, T.M. Atanackovic, An Introduction to Modern Variational Techniques in Mechanics and Engineering. Birkhauser, Boston, 2004.

[27] P. Zhuang, F. Liu, V. Anh, I. Turner, Numerical methods for the variable-order fractional advection-diffusion equation with a nonlinear source term. SIAM J. Numer. Anal. 47 (2009), 1760-1781.

${ }^{1}$ Department of Mechanics, University of Novi Sad

Trg. Dositeja Obradovica 6

21000 - Novi Sad, SERBIA

e-mail: atanackovic@uns.ac.rs_Received: September 14, 2010

${ }^{2}$ Department of Mathematics and Informatics, University of Novi Sad

Trg. Dositeja Obradovica 4

21000 - Novi Sad, SERBIA

e-mail:pilipovic@dmi.uns.ac.rs 